\title{
Predictive Value of Lymphocyte-Related Blood Parameters at the Time Point of Lymphocyte Nadir During Radiotherapy in Breast Cancer
}

This article was published in the following Dove Press journal: OncoTargets and Therapy

\author{
Jiannan Liu (D) ${ }^{1,2}$ \\ Feiyan $\mathrm{Ma}^{3}$ \\ Bing Sun ${ }^{2}$ \\ Yang Cong ${ }^{2}$ \\ Liang Xuan ${ }^{2}$ \\ Qian Wang ${ }^{2}$ \\ Shikai $\mathrm{Wu}^{2}$
}

'Academy of Military Medical Sciences, Beijing 100039, People's Republic of China; ${ }^{2}$ Department of Radiation Oncology, The Fifth Medical Center of Chinese PLA General Hospital (Former 307th Hospital of the PLA), Beijing 10007I, People's Republic of China; ${ }^{3}$ Baoding No.I Central Hospital Affiliated with Chengde Medical College, Baoding City, Hebei Province 071000, People's Republic of China
Correspondence: Shikai Wu

Department of Radiation Oncology, The Fifth Medical Center of Chinese PLA General Hospital (Former 307th Hospital of the PLA), No. 8 East Road, Fengtai District, Beijing 10007I, People's Republic of China

$\mathrm{Tel} / \mathrm{Fax}+861066947621$

Email Skywu4923@sina.com
Purpose: Various reports found a relationship between lymphocyte-related blood parameters (LRBP), including absolute lymphocyte counts (ALC), neutrophil-to-lymphocyte ratio (NLR), and platelet-to-lymphocyte ratio (PLR) and prognosis of breast cancer. Most of the studies focused on LRBP pre-treatment. Seldom have studies focused on LRBP during radiotherapy. We intended to perform a retrospective cohort study on the prognostic value of LRBP at the time point of lowest ALC during radiotherapy for breast cancer.

Patients and methods: A total of 158 female patients were included in radiotherapy group because of the strict limitation standards of complete routine blood test results at pretreatment and pre-operation, and at least once a week during radiotherapy. Besides 221 patients, including the 158 patients of radiotherapy group, were adopted in pre-treatment group and pre-operation group.

Results: ALC and PLR at the time point of lowest ALC during radiotherapy are prognostic predictors of breast cancer, and lower ALC and higher PLR are independent significant predictors of poor DFS. Besides, lower ALC, higher NLR and higher PLR at both pretreatment and pre-operation were found to be independent variables for predicting poor DFS. Conclusion: LRBP at pre-treatment, pre-operation, and during radiotherapy may serve as predictors of outcomes of breast cancer.

Keywords: breast cancer, radiotherapy, disease-free survival, lymphocyte-related blood parameters, lowest absolute lymphocyte counts

\section{Introduction}

Breast cancer is the most common cancer in females around the whole world. ${ }^{1}$ Traditionally, tumor size, presence of lymph node metastasis, clinical stages, pathology grade, and molecular typing are taken as independent prognostic variables in breast cancer. $^{2}$ Nowadays, various studies have shown that different immune states lead to different tumorous outcomes. Increasing evidence suggests that inflammatory factors play an important role in tumor initiation, occurrence, development, recur-

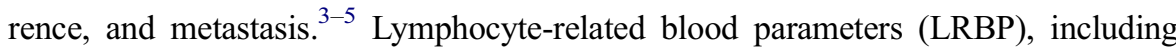
absolute lymphocyte counts (ALC), neutrophil-to-lymphocyte ratio (NLR), and platelet-to-lymphocyte ratio (PLR), representatives of inflammatory factors, can reflect the immune system status. ${ }^{6-8}$ Various reports found a relationship between ALC, NLR, and PLR and prognosis of breast cancer. ${ }^{6-10}$ Most of the studies focused on baseline LRBP. Only a few studies focused on the impact of radiotherapy on LRBP in relation to the prognosis. And in these research, time points of LRBP were 
specifically selected, for example, the time prior to radiotherapy, after 10 fractions of radiotherapy, and at the end of radiotherapy. However, LRBP were varied in different patients during radiotherapy. It seemed that obtaining LRBP during all of the time points, once a week, during radiotherapy and then finding the most representative one to investigate and analyze was a better choice. As radiotherapy leads to lymphopenia, the lowest lymphocyte count during radiotherapy might better represent immune suppression state caused by radiotherapy than lymphocytes of other time points. Therefore, we performed a retrospective cohort study on the prognostic value for breast cancer of LRBP at the time point of lowest ALC during radiotherapy. At the same time, baseline LRBP (before any treatment) and preoperation (before operation) were also collected and analyzed as a comparison with LRBP during radiotherapy and previous relevant studies.

\section{Materials and Methods}

\section{Patients}

This study was reviewed and approved by the ethics committee of The Fifth Medical Center of Chinese PLA General Hospital. Data and research were anonymous to patients. Patients with breast cancer were retrospectively identified using electronic medical records system at The Fifth Medical Center of Chinese PLA General Hospital between January 2007 and December 2014. Prognosis information was obtained by reviewing the electronic medical records, imageological examination and telephonic follow-up. The last followup date was June 2019.

\section{Inclusion and Exclusion}

Inclusion criteria were used to get the study population: 1) diagnosed with invasive ductal carcinoma or invasive lobular carcinoma, with clinical stages among I-III, 2) complete lymphocyte-related blood parameters of baseline, preoperative and radiotherapeutic period, 3) treatment at least with operation and radiotherapy, with or without chemotherapy, 4) negative surgical resection margin status and no residual tumor tissues remaining after operation, 5) the whole treatment completed in our hospital. Exclusion criteria were: patients with 1) incomplete data, 2) clinical stages IV carcinoma, 3) double breast cancer, 4) recurrence or death within 3 months after operation, 5) deaths unrelated to breast cancer, 6) immune dysfunction (such as Human Immunodeficiency Virus infection) or immune hyperfunction (such as autoimmune disease), 7) blood diseases (such as aplastic anemia and leukemia) and 8) other fatal diseases.

\section{Data Collection}

Medical records were reviewed in order to find clinical data of patients, including name, age, sex, pathologic results (histologic type, histological grade, tumor size, and lymph node status, hormone receptor status, and Her-2 receptor status), surgery (breast-conserving surgery and mastectomy), chemotherapy (adjuvant and neoadjuvant chemotherapy), adjuvant radiotherapy, targeted therapy (trastuzumab) and blood routine results, including ALC, absolute neutrophil count (ANC), and absolute platelet count (PLT). The results of routine blood tests were chosen at several points: baseline (before any treatment), pre-operation (before operation), during adjuvant radiotherapy (blood routine test at least once a week). ALC nadir during radiotherapy was defined as the lowest one of all recorded ALC measures during the whole radiotherapy period. DFS was the end point and was defined as the time from surgery to first relapse or death. ER (estrogen receptor) and PR (progesterone receptor) were considered positive if there were at least $1 \%$ positive tumor cells with nuclear staining. Her-2 (human epidermal growth factor receptor 2) was reported positive as the score of $3+$ according to immunohistochemistry or $2+$ with gene amplification verified by florescent in situ hybridization. It was considered as Her-2(-) when the score was $2+$ and the result of florescent in situ hybridization was unavailable. Breast cancer subtypes were defined as follows: Luminal A (ER+ and/or EP+, Her-2-, Ki67<14\%), Luminal B (ER+ and/ or $\mathrm{EP}+$, Her-2-, Ki67 $\geq 14 \%$ ) or (ER+ and/or EP+, Her-2+), Her-2+(ER-, PR-, Her-2+) and TNBC (ER-, PR-, Her-2-). Histopathology results were used to obtain TNM stages according to American Joint Committee on Cancer.

\section{Lymphocyte-Related Blood Parameters (LRBP)}

ALC, ANC, and PLT levels were obtained from routine blood tests, and NLR and PLR were calculated. NLR was calculated as the ratio of absolute neutrophil count to absolute lymphocyte count and PLR was calculated as the ratio of absolute platelet count to absolute lymphocyte count. LRBP included ALC, NLR, and PLR. Three different groups of LRBP were collected and analyzed, baseline group, pre-operation group, and radiotherapy group. In radiotherapy group, all routine blood results during radiotherapy period were compared to find the lowest ALC during radiotherapy. For each patient, a routine blood test 
with the lowest ALC during radiotherapy period was chosen to be analyzed, while the other routine blood results during radiotherapy were not used. According to the Common Terminology Criteria for Adverse Events (version 5.0), lymphopenia was classified as follows: grade 1 , ALC from $0.8 \times 10^{9}$ cells $/ \mathrm{L}$ to $<1.0 \times 10^{9}$ cells $/ \mathrm{L}$; grade 2 , ALC from $0.5 \times 10^{9}$ cells $/ \mathrm{L}$ to $<0.8 \times 10^{9}$ cells $/ \mathrm{L}$; grade 3 , ALC from $0.2 \times 10^{9}$ cells $/ \mathrm{L}$ to $<0.5 \times 10^{9}$ cells $/ \mathrm{L}$; and grade 4 , ALC $<0.2 \times 10^{9}$ cells $/ \mathrm{L}$.

\section{Statistical Analyses}

Statistical analyses were carried out by using SPSS 25.0. Quantitative variables including ALC, NLR, and PLR of the three groups were compared by Wilcoxon rank sum test. The cutoff value was obtained by receiver operating characteristics (ROC) curve and Youden index (Youden index=sensitivity+specificity-1). Kaplan-Meier method was used to calculate the survival curves. Log rank test was used to compare DFS between different groups. To evaluate the relationship between lymphocyte-related blood parameters and DFS, Cox proportional hazards models were used to get prognostic factors and corresponding hazard ratios (HRs) with $95 \%$ confidence intervals (CIs). All statistical tests were two-sided, and $\mathrm{P}<0.05$ was considered statistically significant.

\section{Results}

\section{Patient Characteristics}

A total of 221 female patients were included in the present study (Table 1). The median age at the time of diagnosis was 47 years (range 23-73 years). All patients underwent radical mastectomy $(51,23.1 \%)$ or breast conserving surgery (170, 76.9\%). Chemotherapy drugs included anthracyclines (A), cyclophosphamide (C) and docetaxel (T), and chemotherapy regimens were 4 to 8 courses, every 3 weeks a course, including AC, AT, TC and AC-T. All patients received adjuvant radiotherapy after operation. The radiation dose of postoperative radiotherapy was 50-60 Gy/25-30 fractions (5 days/ week, 5-6 weeks). 171 patients, after breast-conserving surgery, received radiotherapy of $60 \mathrm{~Gy}$ (whole breast irradiation $50 \mathrm{~Gy}+$ tumor bed boost $10 \mathrm{~Gy}$ ). In 51 patients, after mastectomy, 11 patients with tumor stage T4 received radiotherapy of 60 Gy (whole breast irradiation $50 \mathrm{~Gy}+$ tumor bed boost $10 \mathrm{~Gy}$ ), while the other 40 patients received radiotherapy of $50 \mathrm{~Gy}$. All 211 patients had complete routine blood result records of baseline and pre-operation, which were all used for analysis, but only 158 patients' routine blood result records
Table I Baseline Characteristics of Patients

\begin{tabular}{|c|c|}
\hline Parameters & Patients Characteristics, $n(\%)$ \\
\hline$n$ & 221 \\
\hline \multicolumn{2}{|l|}{ Age at diagnosis(years) } \\
\hline$<35$ & $2 \mathrm{I}(9.5 \%)$ \\
\hline$\geq 35$ & $200(90.5 \%)$ \\
\hline \multicolumn{2}{|l|}{ Tumor stage } \\
\hline TI & $49(22.1 \%)$ \\
\hline $\mathrm{T} 2$ & $137(62.0 \%)$ \\
\hline $\mathrm{T} 3$ & $24(10.9 \%)$ \\
\hline $\mathrm{T} 4$ & $\mathrm{II}(5.0 \%)$ \\
\hline \multicolumn{2}{|l|}{ Lymph node status } \\
\hline No & $59(26.7 \%)$ \\
\hline NI & $103(46.6 \%)$ \\
\hline N2 & $28(12.7 \%)$ \\
\hline N3 & $31(14.0 \%)$ \\
\hline \multicolumn{2}{|l|}{ AJCC stage } \\
\hline 1 & $18(8.1 \%)$ \\
\hline ॥ & $125(56.6 \%)$ \\
\hline III & $78(35.3 \%)$ \\
\hline \multicolumn{2}{|l|}{ Tumor grade } \\
\hline GI & $7(3.2 \%)$ \\
\hline G2 & $169(76.5 \%)$ \\
\hline G3 & $36(16.3 \%)$ \\
\hline Unknown & $9(4.0 \%)$ \\
\hline \multicolumn{2}{|l|}{ Molecular subtype } \\
\hline Luminal A & $45(20.4 \%)$ \\
\hline Luminal B & $104(47.1 \%)$ \\
\hline Basal like & $4 I(I 8.5 \%)$ \\
\hline Her2 & $31(14.0 \%)$ \\
\hline \multicolumn{2}{|l|}{ Surgery } \\
\hline BCS & $170(76.9 \%)$ \\
\hline Mastectomy & $5 I(23.1 \%)$ \\
\hline \multicolumn{2}{|l|}{ Chemotherapy } \\
\hline NAC & $128(57.9 \%)$ \\
\hline $\mathrm{AC}$ & $72(32.6 \%)$ \\
\hline $\mathrm{NAC}+\mathrm{AC}$ & $16(7.2 \%)$ \\
\hline None & $5(2.3 \%)$ \\
\hline \multicolumn{2}{|l|}{ Radiotherapy } \\
\hline 50 Gy & $40(18.1 \%)$ \\
\hline $60 \mathrm{~Gy}$ & $|8|(8 \mid .9 \%)$ \\
\hline
\end{tabular}

Abbreviations: AJCC, American Joint Committee on Cancer; BCS, breast-conserving surgery; NAC, neoadjuvant chemotherapy; AC, adjuvant chemotherapy.

during radiotherapy were adopted into research, because for the other 53 patients, there was lack of adequate data during radiotherapy, namely not meeting the standard of routine blood tests, ie, at least once a week during radiotherapy. In 158 patients with lowest ALC during radiotherapy, 122 
patients' lowest ALC occurred in the last week of radiotherapy, 28 occurred in the penultimate week, and 8 occurred in antepenultimate week. The mean ALC, NLR, and PLR of baseline group in 211 patients were $1.86 \pm 0.59\left(\times 10^{9}\right.$ cells/L), $2.18 \pm 0.98$, and $142.11 \pm 51.14$, respectively, and 8 patients had grade 1 lymphopenia, 2 patients had grade 2 lymphopenia, no patients had grade 3 or 4 lymphopenia. The mean ALC, NLR, and PLR of pre-operation group in 211 patients were 1.68 $\pm 0.56\left(\times 10^{9}\right.$ cells/L), $2.07 \pm 1.02$, and $162.59 \pm 70.59$, respectively, and 13 patients had grade 1 lymphopenia, 3 patients had grade 2 lymphopenia, no patients had grade 3 or 4 lymphopenia. The mean ALC, NLR, and PLR of radiotherapy group in 158 patients were $0.86 \pm 0.30\left(\times 10^{9}\right.$ cells/L), $3.16 \pm 1.28$, and $240.61 \pm 102.28$, respectively, and 47 patients had grade 1 lymphopenia, 49 patients had grade 2 lymphopenia, 16 patients had grade 3 lymphopenia, and 2 patients had grade 4 lymphopenia. Pairwise comparison of LRBP among three groups was analyzed by Wilcoxon rank sum test (Table 2). There was a significant difference between each parameter of LRBP in every two groups with $\mathrm{P}<0.05$. The median followup time was 49 months (range 3-133 months). The median DFS was 27 months. Among 221 patients, 49 (22.2\%) patients reached DFS. The number of cases and the percentage of the metastatic and relapse sites were respectively: liver (11, $22.4 \%)$, lymph gland $(11,22.4 \%)$, chest wall $(9,18.4 \%)$, lung $(9,18.4 \%)$, bone $(8,16.3 \%)$, hydrothorax $(3,6.1 \%)$, and brain $(2,4.1 \%)$.

\section{Optimal Cutoff Point of LRBPs for DFS}

The ROC curve of DFS was used to obtain the optimal cutoff points of ALCs, NLRs, and PLRs. The result was shown in Figures 1-3 and Table 3. The optimal cutoff points of ALC, NLR, and PLR of baseline were 1.78 $\left(\times 10^{9}\right.$ cells/L), 2.32 and 176.74 , respectively. And the optimal cutoff points of ALC, NLR, and PLR of preoperation were $1.62\left(\times 10^{9}\right.$ cells $\left./ \mathrm{L}\right), 2.34$ and 186.61, respectively. While the optimal cutoff points of ALC, NLR, and PLR during radiotherapy were $0.96\left(\times 10^{9}\right.$ cells/L), 4.04 and 282.915, respectively. The optimal cutoff points were used to distinguish between high and low of different blood parameters.

\section{Disease-Free Survival by Kaplan-Meier Analysis}

Figures 4-12 showed the Kaplan-Meier curves for DFS. Higher ALC, lower NLR and PLR were associated with improved DFS $(\mathrm{P}<0.05)$, both in baseline group and preoperation group. In radiotherapy group, higher ALC and lower PLR were significantly favorable prognostic factors $(\mathrm{P}<0.05)$, while lower NLR did not reveal the same predictive effect $(\mathrm{P}>0.05)$.

\section{Univariate and Multivariate Analyses for DFS}

We performed univariate and multivariate analyses for DFS in baseline group, pre-operation group, radiotherapy group and baseline characteristic group (Tables 4 and 5). In baseline group, higher ALC, lower NLR, and lower PLR were associated with good prognosis in univariate analysis. Multivariate analyses revealed higher ALC, lower NLR, and lower PLR were independent prognostic factors for improved DFS (respectively: $\mathrm{HR}(95 \% \mathrm{CI}): 0.43(0.24-0.77), \mathrm{P}=0.005 ; \mathrm{HR}$

Table 2 Pairwise Comparison of LRBP by Wilcoxon Rank Sum Test

\begin{tabular}{|c|c|c|c|}
\hline Variable & Mean \pm Standard Deviation & Median & P-value \\
\hline \multicolumn{4}{|c|}{ Baseline vs pre-operation (group) (N=22I) } \\
\hline $\operatorname{ALC}\left(\times 10^{9}\right.$ cells $\left./ \mathrm{L}\right)$ & $1.86 \pm 0.59$ vs $1.68 \pm 0.56$ & 1.83 vs 1.60 & $<0.001$ \\
\hline NLR & $1.68 \pm 0.56$ vs $2.07 \pm 1.02$ & 2.00 vs 1.92 & 0.04 \\
\hline PLR & $|42.1| \pm 5 \mid .14$ vs $162.59 \pm 70.59$ & $\mid 32.50$ vs $\mid 49.64$ & $<0.001$ \\
\hline \multicolumn{4}{|c|}{ Pre-operation vs RT (group) $(\mathrm{N}=158)$} \\
\hline $\operatorname{ALC}\left(\times 10^{9}\right.$ cells $\left./ \mathrm{L}\right)$ & $1.68 \pm 0.56$ vs $0.86 \pm 0.30$ & I.6I vs 0.84 & $<0.001$ \\
\hline NLR & $2.07 \pm 1.02$ vs $3.16 \pm 1.28$ & 1.90 vs 2.99 & $<0.001$ \\
\hline PLR & $162.59 \pm 70.59$ vs $240.61 \pm 102.28$ & $|45.9|$ vs $224.6 \mid$ & $<0.001$ \\
\hline \multicolumn{4}{|c|}{ Baseline vs RT (group) $(\mathrm{N}=158)$} \\
\hline $\operatorname{ALC}\left(\times 10^{9}\right.$ cells $\left./ \mathrm{L}\right)$ & $1.86 \pm 0.59$ vs $0.86 \pm 0.30$ & $\mathrm{I} .80$ vs 0.84 & $<0.001$ \\
\hline NLR & $1.68 \pm 0.56$ vs $3.16 \pm 1.28$ & 2.09 vs 2.99 & $<0.001$ \\
\hline PLR & $|42.1| \pm 5 \mid .14$ vs $240.6 \mid \pm 102.28$ & $\mid 33.40$ vs $224.6 \mid$ & $<0.001$ \\
\hline
\end{tabular}

Abbreviations: ALC, absolute lymphocyte counts; NLR, neutrophil-to-lymphocyte ratio; PLR, platelet-to-lymphocyte ratio; RT, radiation therapy; LRBP, lymphocyte-related blood parameters. 


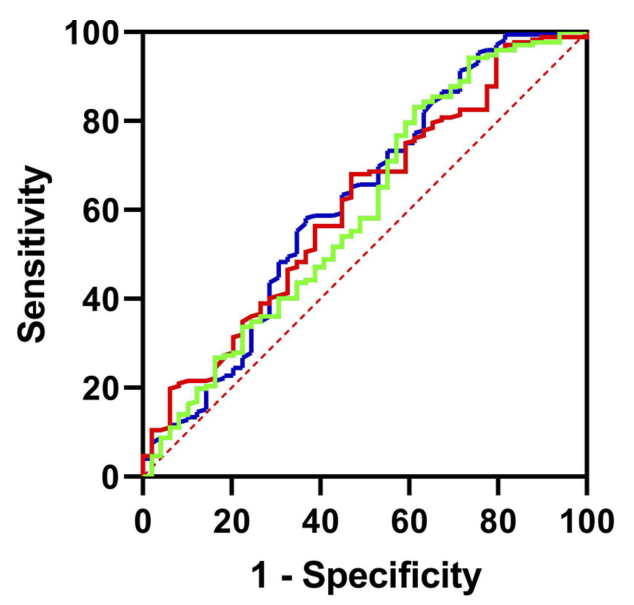

Figure I ROC curves assessing the cutoff points of ALC, NLR and PLR of baseline. Abbreviations: ROC, receiver operating characteristics curve; $A L C$, absolute lymphocyte counts; NLR, neutrophil-to-lymphocyte ratio; PLR, platelet-to-lymphocyte ratio.

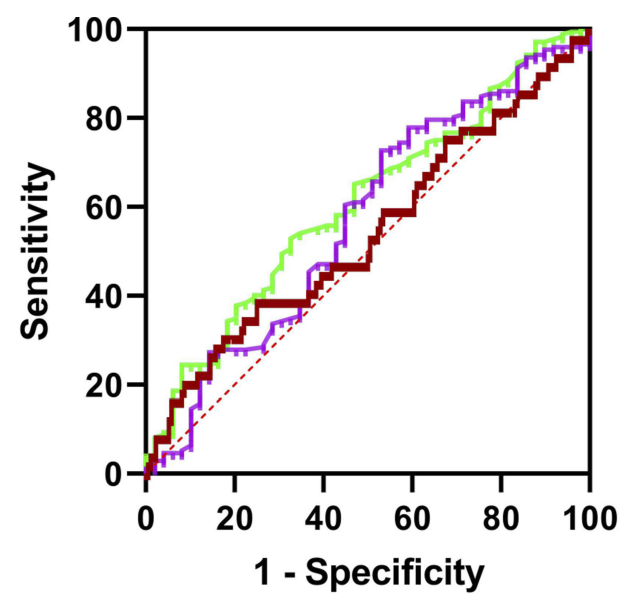

Figure 2 ROC curves assessing the cutoff points of ALC, NLR and PLR of preoperation.

Abbreviations: ROC, receiver operating characteristics curve; ALC, absolute lymphocyte counts; NLR, neutrophil-to-lymphocyte ratio; PLR, platelet-to-lymphocyte ratio.

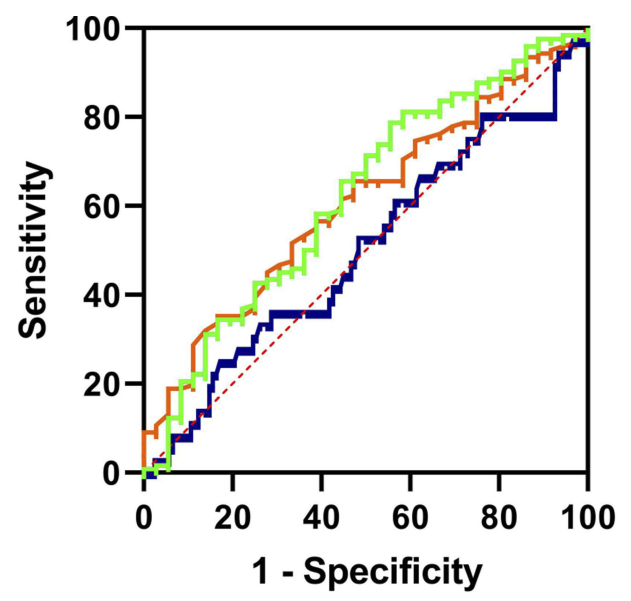

$\longrightarrow$ PLR $\longrightarrow$ NLR $\because \mathrm{ALC}$

Figure 3 ROC curves assessing the cutoff points of ALC, NLR and PLR of radiotherapy.

Abbreviations: ROC, receiver operating characteristics curve; ALC, absolute lymphocyte counts; NLR, neutrophil-to-lymphocyte ratio; PLR, platelet-to-lymphocyte ratio.
Table 3 Relationship Between Blood Parameters and DFS Analyzed by ROC

\begin{tabular}{|l|l|l|l|}
\hline Blood Parameters & $\begin{array}{l}\text { Area } \\
\text { Under the } \\
\text { Curve }\end{array}$ & P-value & $\begin{array}{l}\text { Optimal Cut-off } \\
\text { Point }\end{array}$ \\
\hline Baseline ALC & 0.627 & 0.021 & $1.78\left(\times 10^{9}\right.$ cells/L) \\
Baseline NLR & 0.648 & 0.007 & 2.32 \\
Baseline PLR & 0.569 & 0.210 & 176.74 \\
Preoperative ALC & 0.608 & 0.048 & $1.62\left(\times 10^{9}\right.$ cells/L) \\
Preoperative NLR & 0.574 & 0.176 & 2.34 \\
Preoperative PLR & 0.515 & 0.787 & 186.61 \\
ALC nadir during RT & 0.610 & 0.045 & $0.96\left(\times 10^{9}\right.$ cells/L) \\
NLR during RT & 0.502 & 0.966 & 4.04 \\
PLR during RT & 0.621 & 0.028 & 282.915 \\
\hline
\end{tabular}

Abbreviations: DFS, disease-free survival; ROC, receiver operating characteristics curve; ALC, absolute lymphocyte counts; NLR, neutrophil-to-lymphocyte ratio; PLR, platelet-to-lymphocyte ratio; RT, radiation therapy.

(95\% CI): 2.40(1.35-4.27), $\mathrm{P}=0.003 ; \mathrm{HR}(95 \% \mathrm{CI}): 2.99$ (1.68-5.34), $\mathrm{P}<0.001)$. In pre-operation group, higher ALC, lower NLR, and lower PLR had the similar independent predictive effect for DFS (respectively: $\mathrm{HR}(95 \% \mathrm{CI}): 0.42(0.23-$ 0.76), $\mathrm{P}=0.004$; $\mathrm{HR}(95 \% \mathrm{CI}): 0.42(0.23-0.76), \mathrm{P}=0.008 ; \mathrm{HR}$ (95\% CI): 2.01(1.12-3.60), $\mathrm{P}=0.020$ ). In radiotherapy group, higher ALC and lower PLR were found to be independent variables for predicting favorable DFS in multivariable analysis (respectively: $\mathrm{HR}(95 \% \mathrm{CI}): 0.37(0.15-0.89), \mathrm{P}=0.027$; HR (95\% CI): $3.40(1.72-6.72), \mathrm{P}<0.001$ ), while, NLR did not show a significant association with patients' DFS both in univariate and multivariate analyses. In multivariate analyses of three groups, co-variables included age, TNM stage, hormone receptor status, Her-2 status, tumor grade, and Ki67. In baseline characteristic group, age, TNM stage, hormone receptor status, Her-2 status, tumor grade, and Ki67 were included in analysis. High clinical stage, negative hormone receptor,

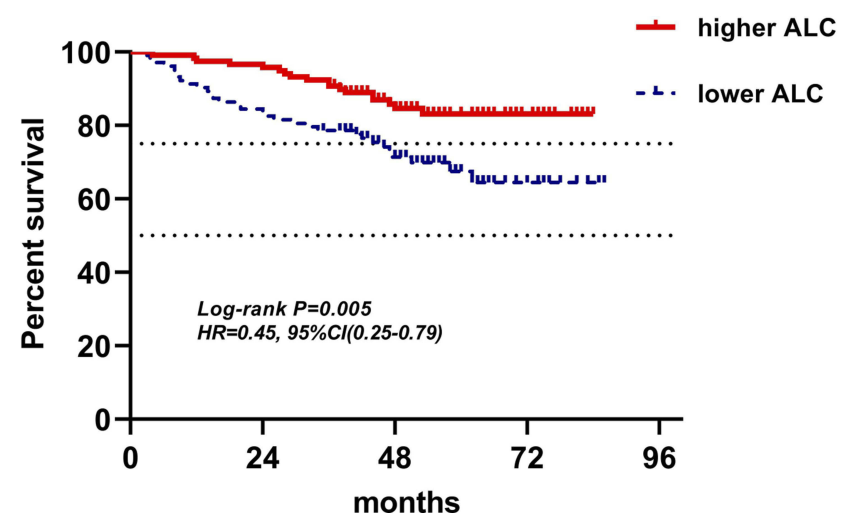

Figure 4 Kaplan-Meier curves of low and high ALC of baseline for DFS. Abbreviations: ALC, absolute lymphocyte counts; DFS, disease-free survival. 


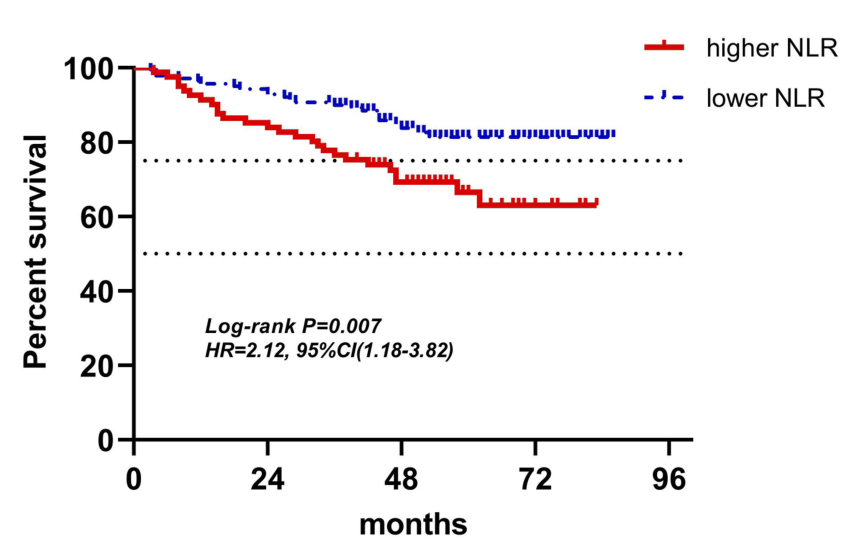

Figure 5 Kaplan-Meier curves of low and high NLR of baseline for DFS. Abbreviations: NLR, neutrophil-to-lymphocyte ratio; DFS, disease-free survival.

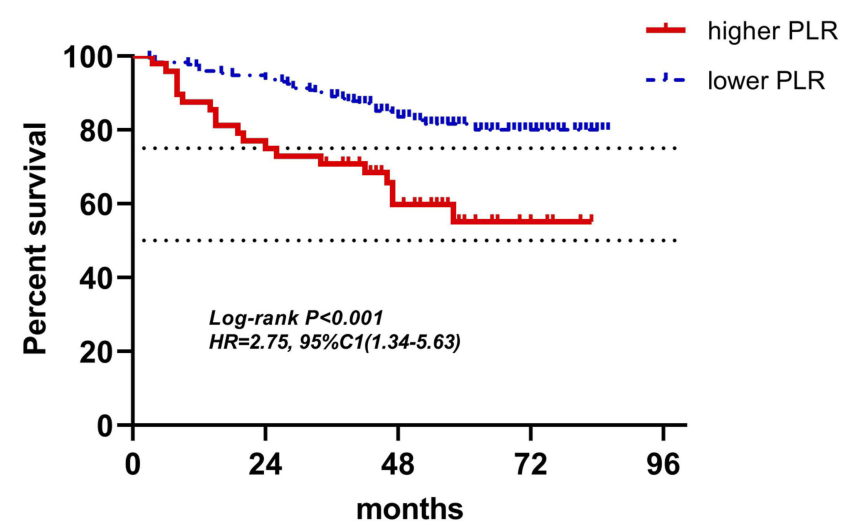

Figure 6 Kaplan-Meier curves of low and high PLR of baseline for DFS. Abbreviations: PLR, platelet-to-lymphocyte ratio; DFS, disease-free survival.

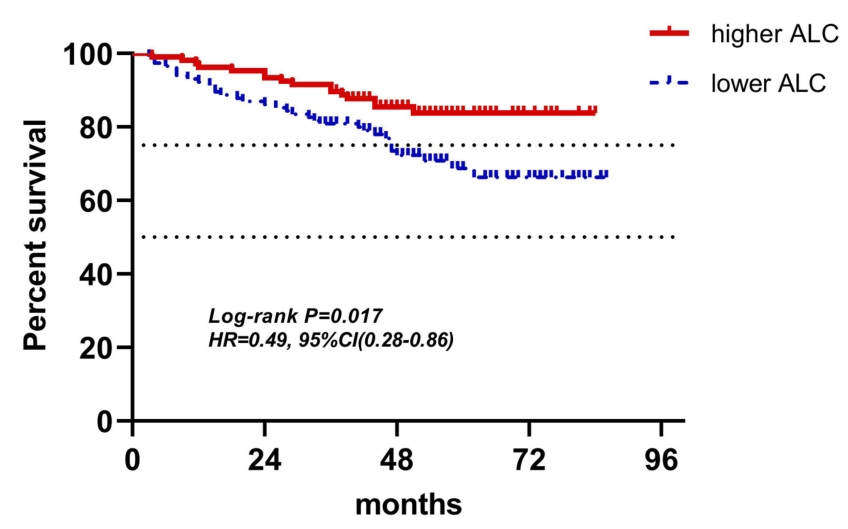

Figure 7 Kaplan-Meier curves of low and high ALC of pre-operation for DFS Abbreviations: ALC, absolute lymphocyte counts; DFS, disease-free survival.

high tumor grade and high Ki67 index were calculated as predictors of poor survival in univariate analysis. Multivariate analysis also revealed high clinical stage, negative hormone receptor, high tumor grade and high Ki67 index were independent prognostic factors for poor DFS

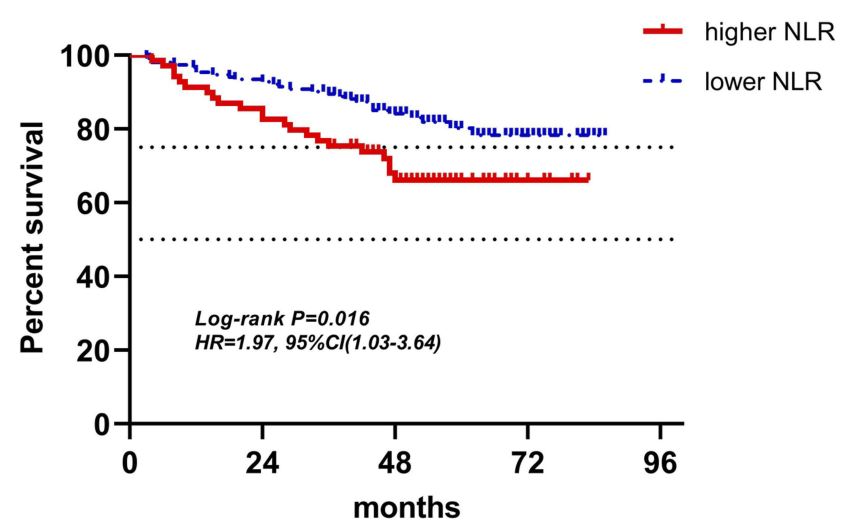

Figure 8 Kaplan-Meier curves of low and high NLR of pre-operation for DFS. Abbreviations: NLR, neutrophil-to-lymphocyte ratio; DFS, disease-free survival.

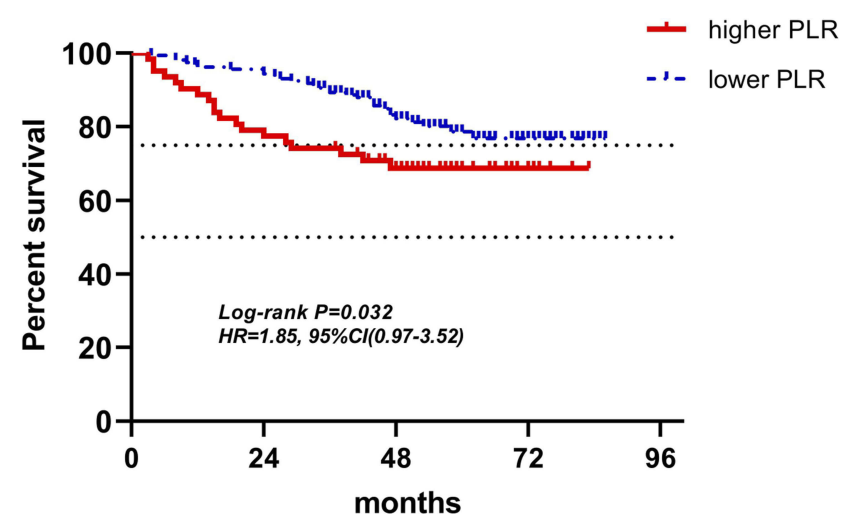

Figure 9 Kaplan-Meier curves of low and high PLR of pre-operation for DFS. Abbreviations: PLR, platelet-to-lymphocyte ratio; DFS, disease-free survival.

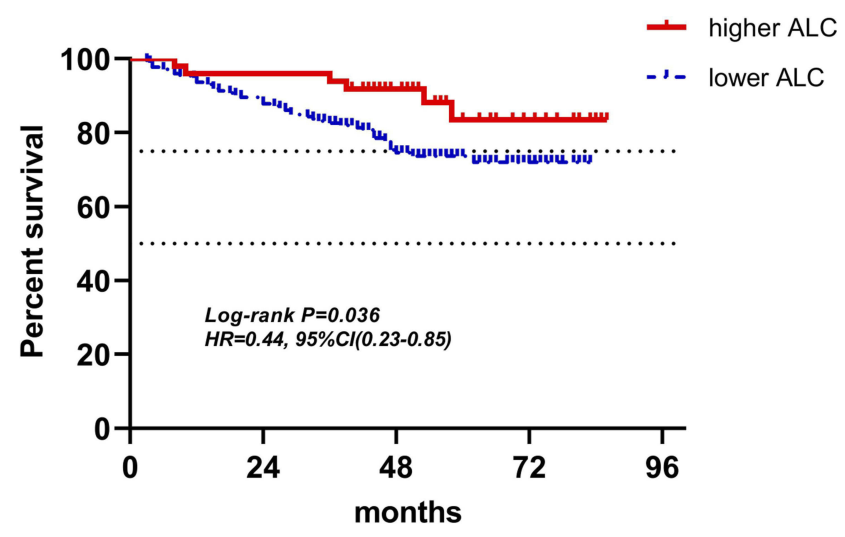

Figure 10 Kaplan-Meier curves of low and high ALC of radiotherapy for DFS. Abbreviations: ALC, absolute lymphocyte counts; DFS, disease-free survival.

(respectively: $\mathrm{HR}(95 \% \mathrm{CI}): 2.28(1.29-4.01), \mathrm{P}=0.004$; HR (95\% CI): $0.53(0.35-0.82), \mathrm{P}=0.004 ; \mathrm{HR}(95 \% \mathrm{CI}): 1.89$ (1.20-2.98), $\quad \mathrm{P}=0.006 ; \quad \mathrm{HR}(95 \% \quad \mathrm{CI}): \quad 3.07(1.27-7.39)$, $\mathrm{P}=0.013$ ). However, no obvious correlation was seen between age, Her-2 status, and prognosis. 


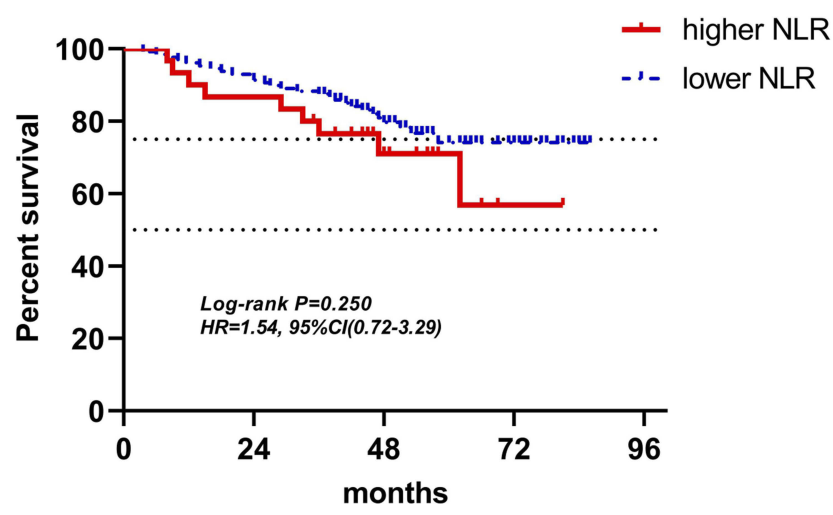

Figure I I Kaplan-Meier curves of low and high NLR of radiotherapy for DFS. Abbreviations: NLR, neutrophil-to-lymphocyte ratio; DFS, disease-free survival.

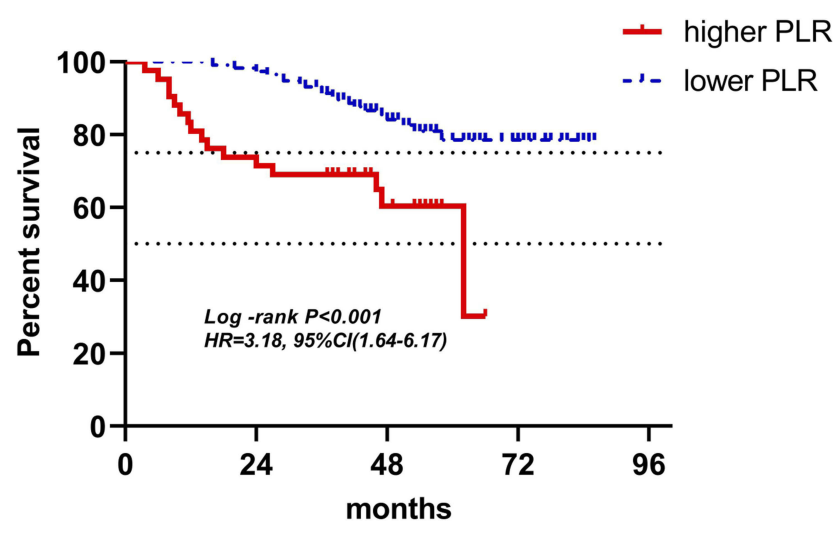

Figure 12 Kaplan-Meier curves of low and high PLR of radiotherapy for DFS. Abbreviations: PLR, platelet-to-lymphocyte ratio; DFS, disease-free survival.

\section{Discussion}

We performed this retrospective cohort study on the prognostic value for breast cancer of LRBP at the time point of lowest ALC during radiotherapy. At the same time, LRBP of baseline and pre-operation were also collected and analyzed as a comparison with LRBP during radiotherapy and previous related studies. Various reports found a relationship between ALC, NLR and PLR and prognosis of breast cancer, most of the studies focusing on LRBP of baseline. ${ }^{6-10}$ However, only a few studies focused on the impact of radiotherapy on LRBP in relation to prognosis. And in these research, time points of LRBP were artificial selection, and most of them were before or after radiotherapy. ${ }^{11,12}$ It seemed that obtaining LRBP of all time points, once a week, during radiotherapy and then finding the most representative one to investigate and analyze was a better choice. As radiotherapy leads to lymphopenia, the lowest lymphocyte count during radiotherapy might better represent immune suppression state caused by radiotherapy than lymphocytes during other time points, so LRBP at the time point of lowest ALC during radiotherapy was chosen in our study, which was different from the previous research.

Most similar studies focused on LRBP of one specific point during therapy, for example, before treatment, before operation, on a certain time of chemotherapy or radiotherapy. Our study selected data from three different periods of therapy, including baseline, pre-operation and radiotherapy. Variation of LRBP was seen with the change of treatment methods and treatment time, but we could see the relatively stable predictive function of LRBP for prognostic value in different treatment periods, except for NLR at the time of lowest ALC during radiotherapy.

Moreover, although most patients undergoing radiotherapy were required to have routine blood test once a week to evaluate their physical condition, as a matter of fact, only a few patients received routine blood test once a week, because most of them were outpatients, hard to manage, and compared to chemotherapy with more systemic side effects, the side effects of radiotherapy were mainly local, and systemic reactions were mild. ${ }^{13,14}$ Most patients did not go for routine blood test once a week to evaluate their physical condition arranged by the doctors. So, it was not easy to get a record of patients with routine blood tests once a week during radiotherapy.

Lymphocytes play an important role in cellular immunity against malignant cells. ${ }^{3,15}$ Various studies have proven that low ALC is related to worse prognosis in different cancers, including head and neck cancer, esophageal cancer, rectal cancer, non-small cell lung cancer, small-cell lung cancer, and breast cancer. ${ }^{16-20}$ Neutrophils were considered to have a relationship with tumor progression and metastasis by releasing immune mediators, such as interleukin-8, neutrophil elastase, and matrix metalloproteinase- $9 .{ }^{21-23}$ Platelets were found to be associated with promoting angiogenesis, releasing adhesion molecules and growth factors, and modifying the extracellular matrix, and therefore having a correlation with progression and metastasis of tumors. ${ }^{24-26}$ Previous studies have shown that both higher NLR and higher PLR have a relationship with poor prognosis in breast cancer, however, the underlying mechanisms are still unclear. It is now thought that higher NLR and higher PLR mean increased neutrophils and platelets and/or decreased lymphocytes, which suggests poor survival in breast cancer.

In the radiotherapy group, because the inclusion criteria included routine blood test at least once a week during 
Table 4 Univariate and Multivariate Analyses of Lymphocyte-Related Blood Parameters (LRBP) for DFS

\begin{tabular}{|c|c|c|c|c|c|c|}
\hline \multirow[t]{2}{*}{$\operatorname{LRBP}(n)$} & \multirow[t]{2}{*}{ Higher n(\%) } & \multirow[t]{2}{*}{ Lower n(\%) } & \multicolumn{2}{|c|}{ Univariate Analyses } & \multicolumn{2}{|c|}{ Multivariate Analyses } \\
\hline & & & $\operatorname{HR}(95 \% \mathrm{Cl})$ & P-value & HR(95\% Cl) & P-value \\
\hline \multicolumn{7}{|l|}{ Baseline LRBP } \\
\hline $\operatorname{ALC}(221)$ & $118(53.4 \%)$ & $103(46.6 \%)$ & $0.45(0.25-0.79)$ & 0.005 & $0.43(0.24-0.77)$ & 0.005 \\
\hline $\operatorname{NLR}(22 I)$ & $81(36.7 \%)$ & $140(63.3 \%)$ & $2.12(1.18-3.82)$ & 0.007 & $2.40(1.35-4.27)$ & 0.003 \\
\hline $\operatorname{PLR}(22 I)$ & $48(21.7 \%)$ & $173(78.3 \%)$ & $2.75(1.34-5.63)$ & $<0.001$ & $2.99(1.68-5.34)$ & $<0.001$ \\
\hline \multicolumn{7}{|c|}{ Preoperative LRBP } \\
\hline $\operatorname{ALC}(22 \mathrm{I})$ & $106(48.0 \%)$ & $115(52.0 \%)$ & $0.49(0.28-0.86)$ & 0.017 & $0.42(0.23-0.76)$ & 0.004 \\
\hline $\operatorname{NLR}(22 \mathrm{I})$ & $69(31.2 \%)$ & $152(68.8 \%)$ & $1.97(1.03-3.64)$ & 0.016 & $2.18(1.23-3.87)$ & 0.008 \\
\hline $\operatorname{PLR}(221)$ & $159(71.9 \%)$ & $62(28.1 \%)$ & $1.85(0.97-3.52)$ & 0.032 & $2.01(1.12-3.60)$ & 0.020 \\
\hline \multicolumn{7}{|l|}{ LRBP during RT } \\
\hline ALC (158) & $49(31.0 \%)$ & $109(69.0 \%)$ & $0.44(0.23-0.85)$ & 0.036 & $0.37(0.15-0.89)$ & 0.027 \\
\hline NLR (158) & $30(19.0 \%)$ & $128(81.0 \%)$ & I.54(0.72-3.29) & 0.250 & $1.55(0.72-3.33)$ & 0.265 \\
\hline PLR (I58) & $42(26.6 \%)$ & $116(73.4 \%)$ & $3.18(1.64-6.17)$ & $<0.001$ & $3.40(1.72-6.72)$ & $<0.001$ \\
\hline
\end{tabular}

Abbreviations: DFS, disease-free survival; ALC, absolute lymphocyte counts; NLR, neutrophil-to-lymphocyte ratio; PLR, platelet-to-lymphocyte ratio; RT, radiation therapy; LRBP, lymphocyte-related blood parameters; HR, hazard ratio; Cl, confidence interval.

Table 5 Univariate and Multivariate Analyses of Clinical and Pathological Variables for DFS

\begin{tabular}{|c|c|c|c|c|}
\hline \multirow[t]{2}{*}{ Variable } & \multicolumn{2}{|c|}{ Univariate Analyses } & \multicolumn{2}{|c|}{ Multivariate Analyses } \\
\hline & HR(95\% Cl) & P-value & HR(95\% Cl) & P-value \\
\hline $\begin{array}{l}\text { Age } \\
(<35 \text { vs } \geq 35)\end{array}$ & $0.75(0.3|-| .75)$ & 0.499 & $0.70(0.30-1.67)$ & 0.424 \\
\hline $\begin{array}{l}\text { TNM stage } \\
(I+I I \text { vs III) }\end{array}$ & $2.35(1.34-4.12)$ & 0.003 & $2.28(1.29-4.01)$ & 0.004 \\
\hline $\begin{array}{l}\text { Hormone receptor status } \\
(E R+\text { and/or PR+ vs ER- and PR-) }\end{array}$ & $0.40(0.27-0.59)$ & $<0.001$ & $0.53(0.35-0.82)$ & 0.004 \\
\hline $\begin{array}{l}\text { Her-2 status } \\
\text { (Her-2+ vs Her-2-) }\end{array}$ & $0.77(0.40-1.48)$ & 0.439 & $0.63(0.32-1.23)$ & 0.178 \\
\hline $\begin{array}{l}\text { Tumor grade } \\
(G I+G 2 \text { vs } G 3)\end{array}$ & $2.58(1.68-3.96)$ & $<0.001$ & $1.89(1.20-2.98)$ & 0.006 \\
\hline $\begin{array}{l}\text { Ki67 } \\
(14 \%<v s \geq 14 \%)\end{array}$ & $3.14(1.34-7.39)$ & 0.009 & $3.07(1.27-7.39)$ & 0.013 \\
\hline
\end{tabular}

Abbreviations: DFS, disease-free survival; ER, estrogen receptor; PR, progesterone receptor; Her-2, human epidermal growth factor receptor 2; HR, hazard ratio; Cl, confidence interval.

radiotherapy, only 158 patients' LRBP were adopted into research. As mentioned previously, it was not easy to find a patient with routine blood test once a week during radiotherapy, so despite the fact that only 158 patients' radiotherapy data were involved in analysis, a small quantity as it may be, it was still very valuable. In multivariate analyses for DFS, higher ALC and lower PLR were found to be independent variables for predicting favorable outcomes (respectively: $\operatorname{HR}(95 \% \mathrm{CI}): 0.37(0.15-0.89), \mathrm{P}=0.027 ; \operatorname{HR}(95 \%$
CI): $3.40(1.72-6.72), \mathrm{P}<0.001$ ), which meant that ALC and PLR at the time point of lowest ALC during radiotherapy were associated with survival. While, NLR did not show a significant association with patients' DFS both in univariate and multivariate analyses. But in NLR's Kaplan-Meier curves for DFS, two curves represented higher NLR and lower NLR was separate, higher NLR indicated poor DFS. Besides, in univariate and multivariate analyses, hazard ratios with $95 \%$ confidence interval of higher NLR were 
1.54(0.72-3.29), and 1.54(0.72-3.29), respectively, both greater than one, meaning the higher the NLR, the worse the outcomes. P-values of NLR for DFS in univariate and multivariate analyses were 0.250 and 0.265 , both greater than 0.05 , but when considering small sample size, higher NLR may be associated with poor DFS in a large sample analysis. Which, however, was just speculation, and needs to be proven. As lymphocytes were considered to be the most sensitive cells to radiotherapy followed by neutrophils, monocytes, then platelets and erythrocytes, the counts of lymphocytes decreased obviously during the process of radiotherapy, and eventually, among 158 patients of radiotherapy group, at the point of lowest ALC during radiotherapy, $72.2 \%$ patients had lymphopenia, however, in 221 patients of baseline and pre-operation groups, only $4.5 \%$ and $7.2 \%$ patients had lymphopenia, respectively. ${ }^{27}$ The mean ALC, NLR, and PLR of radiotherapy group in 158 patients were $0.86 \pm 0.30(\times 109$ cells/L), $3.16 \pm 1.28$, and $240.61 \pm 102.28$, respectively. The difference of each parameter of LRBP between radiotherapy group and either baseline group or pre-operation group was significant with $\mathrm{P}<0.001$ by Wilcoxon rank sum test. In 158 patients with lowest ALC during radiotherapy, 122 patients' lowest ALC occurred in the last week of radiotherapy, 28 occurring in the penultimate week, and 8 occurring in antepenultimate week. From $77.2 \%$ patients' lowest ALC occurring in the last week of radiotherapy, it could be seen that total radiotherapy dose was the dominant factor in lymphopenia. However, $22.8 \%$ patients' lowest ALC did not occur in the last week, which illustrated that other factors affected the result of lymphopenia, for example, individual difference of radiosensitivity, some patients' lymphocytes having hypersensitivity to radiotherapy and rapidly decreasing, until reaching the nadir, then increasing slowly during radiotherapy.

In baseline and pre-operation groups, ALC, NLR and PLR had similar predictive values for prognosis. Higher ALC, lower NLR, and lower PLR were independent prognostic factors with good prognosis both in baseline group (respectively: $\mathrm{HR}(95 \% \mathrm{CI}): 0.43(0.24-0.77), \mathrm{P}=0.005$; HR (95\% CI): 2.40(1.35-4.27), P=0.003; HR(95\% CI): 2.99 (1.68-5.34), $\mathrm{P}<0.001)$ and pre-operation group (respectively: $\operatorname{HR}(95 \% \mathrm{CI}): 0.42(0.23-0.76), \mathrm{P}=0.004 ; \mathrm{HR}(95 \%$ CI): $0.42(0.23-0.76), \mathrm{P}=0.008$; HR(95\% CI): $2.01(1.12$ 3.60), $\mathrm{P}=0.020$ ). It illustrated that LRBP could reflect the condition of immune status at the time points of baseline and pre-operation, and immune status, as is well-known, has a close relationship with tumor progression and metastasis. ${ }^{3,5,15}$ Similar outcomes were found in previous studies investigating the correlation between LRBP of both baseline and pre-operation and breast cancer prognosis. ${ }^{6,8,10,28-30}$ The mean ALC, NLR, and PLR of baseline group were $1.86 \pm 0.59\left(\times 10^{9}\right.$ cells $\left./ \mathrm{L}\right), 2.18 \pm 0.98$, and 142.11 \pm 51.14 , respectively, and the mean ALC, NLR, and PLR of pre-operation group in 211 patients were 1.68 $\pm 0.56 \quad\left(\times 10^{9}\right.$ cells $\left./ \mathrm{L}\right), \quad 2.07 \pm 1.02$, and $162.59 \pm 70.59$, respectively. The difference between each parameter of LRBP in two groups was significant with $\mathrm{P}<0.05$, which meant that lymphocyte level decreased from baseline to pre-operation. It was probable that 128 patients received neoadjuvant chemotherapy, which suppressed the function of bone marrow and induced lymphopenia.

In baseline characteristic group, age, TNM stage, hormone receptor status, Her-2 status, tumor grade, and Ki67 were included in analysis. High clinical stage, negative hormone receptor, high tumor grade and high Ki67 index were calculated as predictors of poor survival in both univariate and multivariate analyses, which matched what has been proven. No obvious correlation was seen between age, Her-2 status and prognosis. Among 221 patients, only 21 patients' age were under 35, so the result of age might be different with a bigger sample size. The reasons for result of Her-2 status are complicated, it may relate to whether they received standard targeted therapy, whether they had negative hormone receptor, which can lead to different outcomes of prognosis.

This small size, retrospective research had certain inherent limitations. The number of patients were not big, all from one institution, only 221 patients were included in the study, and only 158 patients' LRBP were adopted in analysis in radiotherapy group. However, the reason for the small size was that we strictly limited standards in that patients had to have complete routine blood test results of all time points during treatment, including baseline, pre-operation and radiotherapy period. Further, 63 patients of the 211 were excluded from analysis in the radiotherapy group because they had incomplete routine blood test results, ie, they did not go for a blood test at least once a week during radiotherapy. As mentioned previously, it was not easy to get a record of patients with routine blood tests once a week during radiotherapy. Despite the small number of the study, it has provided unstudied content of the prognostic value for breast cancer of LRBP at the time point of lowest ALC during radiotherapy.

\section{Conclusion}

Our study suggests that ALC and PLR at the time point of lowest ALC during radiotherapy are prognostic predictors 
of breast cancer, and lower ALC and higher PLR are independent significant predictors of poor DFS. Besides, lower ALC, higher NLR and higher PLR at both baseline and pre-operation were found to be independent variables for predicting poor DFS, as shown in previous studies.

\section{Disclosure}

The authors report no conflicts interest in this work.

\section{References}

1. Winters S, Martin C, Murphy D, Shokar NK. Breast cancer epidemiology, prevention, and screening. Prog Mol Biol Transl Sci. 2017;151:1-32.

2. Tao Z, Shi A, Lu C, Song T, Zhang Z, Zhao J. Breast cancer: epidemiology and etiology. Cell Biochem Biophys. 2015;72(2):333338. doi:10.1007/s12013-014-0459-6

3. Romaniuk A, Lyndin M. Immune microenvironment as a factor of breast cancer progression. Diagn Pathol. 2015;10:79. doi:10.1186/ s13000-015-0316-y

4. Disis ML, Stanton SE. Immunotherapy in breast cancer: an introduction. Breast. 2018;37:196-199. doi:10.1016/j.breast.2017.01.013

5. Yu LY, Tang J, Zhang CM, et al. New immunotherapy strategies in breast cancer. Int $J$ Environ Res Public Health. 2017;14:1. doi:10.3390/ijerph14010068

6. Asano Y, Kashiwagi S, Onoda N, et al. Platelet-lymphocyte ratio as a useful predictor of the therapeutic effect of neoadjuvant chemotherapy in breast cancer. PLoS One. 2016;11(7):e0153459. doi:10.1371/ journal.pone. 0153459

7. Vicente Conesa MA, Garcia-Martinez E, Gonzalez Billalabeitia E, et al. Predictive value of peripheral blood lymphocyte count in breast cancer patients treated with primary chemotherapy. Breast. 2012;21 (4):468-474. doi:10.1016/j.breast.2011.11.002

8. Chen Y, Chen K, Xiao X, et al. Pretreatment neutrophil-to-lymphocyte ratio is correlated with response to neoadjuvant chemotherapy as an independent prognostic indicator in breast cancer patients: a retrospective study. BMC Cancer. 2016;16:320. doi:10.1186/s12885-0162352-8

9. Ni XJ, Zhang XL, Ou-Yang QW, et al. An elevated peripheral blood lymphocyte-to-monocyte ratio predicts favorable response and prognosis in locally advanced breast cancer following neoadjuvant chemotherapy. PLoS One. 2014;9(11):e111886. doi:10.1371/journal. pone. 0111886

10. Ou Q, Cheng J, Zhang L, Wang H, Wang W, Ma Y. The prognostic value of pretreatment neutrophil-to-lymphocyte ratio in breast cancer: deleterious or advantageous? Tumour Biol. 2017;39(6):1010428317706214. doi:10.1177/1010428317706214

11. Cho O, Chun M, Kim SW, Jung YS, Yim H. Lymphopenia as a potential predictor of ipsilateral breast tumor recurrence in early breast cancer. Anticancer Res. 2019;39(8):4467-4474. doi:10.21873/ anticanres. 13620

12. Jensen GL, Blanchard P, Gunn GB, et al. Prognostic impact of leukocyte counts before and during radiotherapy for oropharyngeal cancer. Clin Transl Radiat Oncol. 2017;7:28-35. doi:10.1016/j. ctro.2017.09.008

13. Kanegasaki S, Yamashita T, Tsuchiya T. Reduced number of lymphocytes by X-ray irradiation: a problem in a combination therapy trial that elicits the abscopal effect in preclinical studies using electron beam irradiation. Cureus. 2019;11(2):e4142.
14. Herrera FG, Bourhis J, Coukos G. Radiotherapy combination opportunities leveraging immunity for the next oncology practice. $C A$ Cancer J Clin. 2017;67(1):65-85. doi:10.3322/caac.21358

15. Bates JP, Derakhshandeh R, Jones L, Webb TJ. Mechanisms of immune evasion in breast cancer. BMC Cancer. 2018;18(1):556. doi:10.1186/s12885-018-4441-3

16. Campian JL, Sarai G, Ye X, Marur S, Grossman SA. Association between severe treatment-related lymphopenia and progression-free survival in patients with newly diagnosed squamous cell head and neck cancer. Head Neck. 2014;36(12):1747-1753. doi:10.1002/hed. v36.12

17. Remark R, Becker C, Gomez JE, et al. The non-small cell lung cancer immune contexture. A major determinant of tumor characteristics and patient outcome. Am J Respir Crit Care Med. 2015;191 (4):377-390. doi:10.1164/rccm.201409-1671PP

18. Suzuki R, Wei X, Allen PK, Cox JD, Komaki R, Lin SH. Prognostic significance of total lymphocyte count, neutrophil-to-lymphocyte ratio, and platelet-to-lymphocyte ratio in limited-stage small-cell lung cancer. Clin Lung Cancer. 2019;20(2):117-123. doi:10.1016/j. cllc.2018.11.013

19. Deng W, Xu C, Liu A, et al. The relationship of lymphocyte recovery and prognosis of esophageal cancer patients with severe radiationinduced lymphopenia after chemoradiation therapy. Radiother Oncol. 2019;133:9-15. doi:10.1016/j.radonc.2018.12.002

20. Campian JL, Ye X, Sarai G, Herman J, Grossman SA. Severe treatmentrelated lymphopenia in patients with newly diagnosed rectal cancer. Cancer Invest. 2018;36(6):356-361. doi:10.1080/07357907.2018. 1499028

21. Liang W, Ferrara N. The complex role of neutrophils in tumor angiogenesis and metastasis. Cancer Immunol Res. 2016;4(2):8391. doi:10.1158/2326-6066.CIR-15-0313

22. Labelle M, Begum S, Hynes RO. Direct signaling between platelets and cancer cells induces an epithelial-mesenchymal-like transition and promotes metastasis. Cancer Cell. 2011;20(5):576-590. doi:10.1016/j.ccr.2011.09.009

23. Houghton AM, Rzymkiewicz DM, Ji H, et al. Neutrophil elastasemediated degradation of IRS-1 accelerates lung tumor growth. Nat Med. 2010;16(2):219-223. doi:10.1038/nm.2084

24. Swierczak A, Mouchemore KA, Hamilton JA, Anderson RL. Neutrophils: important contributors to tumor progression and metastasis. Cancer Metastasis Rev. 2015;34(4):735-751. doi:10.1007/ s10555-015-9594-9

25. Menter DG, Kopetz S, Hawk E, et al. Platelet "first responders" in wound response, cancer, and metastasis. Cancer Metastasis Rev. 2017;36(2):199-213. doi:10.1007/s10555-017-9682-0

26. Kanikarla-Marie P, Lam M, Menter DG, Kopetz S. Platelets, circulating tumor cells, and the circulome. Cancer Metastasis Rev. 2017;36 (2):235-248. doi:10.1007/s10555-017-9681-1

27. Zachariah B, Jacob SS, Gwede C, et al. Effect of fractionated regional external beam radiotherapy on peripheral blood cell count. Int $J$ Radiat Oncol Biol Phys. 2001;50(2):465-472. doi:10.1016/S03603016(00)01587-X

28. Hong J, Mao Y, Chen X, et al. Elevated preoperative neutrophil-tolymphocyte ratio predicts poor disease-free survival in Chinese women with breast cancer. Tumour Biol. 2016;37(3):4135-4142. doi:10.1007/s13277-015-4233-1

29. Hu RJ, Liu Q, Ma JY, Zhou J, Liu G. Preoperative lymphocyte-tomonocyte ratio predicts breast cancer outcome: a meta-analysis. Clin Chim Acta. 2018;484:1-6. doi:10.1016/j.cca.2018.05.031

30. Yao M, Liu Y, Jin H, et al. Prognostic value of preoperative inflammatory markers in Chinese patients with breast cancer. Onco Targets Ther. 2014;7:1743-1752. doi:10.2147/OTT.S69657 


\section{Publish your work in this journal}

OncoTargets and Therapy is an international, peer-reviewed, open access journal focusing on the pathological basis of all cancers, potential targets for therapy and treatment protocols employed to improve the management of cancer patients. The journal also focuses on the impact of management programs and new therapeutic agents and protocols on patient perspectives such as quality of life, adherence and satisfaction. The manuscript management system is completely online and includes a very quick and fair peer-review system, which is all easy to use. Visit http://www.dovepress.com/ testimonials.php to read real quotes from published authors.

Submit your manuscript here: https://www.dovepress.com/oncotargets-and-therapy-journal 